\title{
Magnetic Resonance Imaging of time-varying magnetic fields from therapeutic devices
}

\author{
Luis Hernandez-Garcia, Vivek Bhatia, Krishan Prem-Kumar, and Magnus Ulfarsson
}

\begin{abstract}
While magnetic resonance imaging of static magnetic fields generated by external probes has been previously demonstrated, there is an unmet need to image time-varying magnetic fields, such as those generated by transcranial magnetic stimulators or radiofrequency hyperthermia probes. A method to image such time-varying magnetic fields is introduced in this work. This article presents the theory behind the method and provides proof of concept by imaging time-varying magnetic fields generated by a figure-eight coil inside simple phantoms over a range of frequencies and intensities, using a 7T small animal MRI scanner. The method is able to reconstruct the three-dimensional components of the oscillating magnetic field vector.
\end{abstract}

\section{Introduction}

Magnetic resonance imaging (MRI) is exquisitely sensitive to magnetic field perturbations. Indeed, a great deal effort has been devoted to overcoming the effects of those perturbations on the images. On the bright side, that sensitivity has also been leveraged to map the quasistatic magnetic fields generated by therapeutic devices, such as transcranial magnetic stimulation (TMS) probes. This application is very compelling, as it would greatly facilitate the design testing and validation of new probes as well as targeting. While TMS pulses are not static, they are of a low enough frequency that they can be easily approximated by scaling a small static magnetic field produced by the same TMS by the driving current's waveform(1-3). This approximation breaks down as the frequency of the field increases and secondary (induced) fields become more prominent, so it may not be suitable for the case of thermal ablation RF therapy (4) or other devices.

In that case, magnetic fields and specific absorption rate (SAR) maps can be calculated for the purposes of device safety by computational modeling over the entire frequency spectrum, if the spatial distribution of the electrical properties (permittivity, conductivity, ..., etc.) of the tissue is known. Thus, it may prove advantageous to image the fields directly, as it circumvents making additional measurements and assumptions. It should be noted that if the device operates at the resonant frequency of the spins, one could map it by measuring the flip angles produced by its field, but this method is restricted to that specific resonant frequency and is appropriate mostly for the RF probes employed in MRI.

The aim of the present work is to present proof of concept of a simple method to image dynamic magnetic fields induced in the body by an electromagnetic probe (therapeutic or otherwise) driven by an arbitrary known waveform. Static magnetic field imaging has been demonstrated by observing phase shifts in the signal resulting from changes in echo time (13,5). Similarly, the Bloch-Siegert effect has been also leveraged to map the fields produced the imaging RF coils, but this is limited to the frequency range of the imaging coil (6) and

Contact: Luis Hernandez-Garcia, Ph.D., FMRI Laboratory, 2360 Bonisteel Blvd., Ann Arbor, MI 48109-2108, Tel: (734) 763 9254, Fax: (734) 9364128 , hernan@umich.edu. 
produces only the component of the field that is collinear with the RF coil's main axis. Stimulus induced resonant saturation (SIRS) can be used to map narrow band oscillating fields but requires transmission of a secondary spin-locking pulse using additional hardware (7). The approach that we present in this article requires no additional hardware and allows mapping the spatial distribution of all three Cartesian components of a magnetic vector field oscillating with an arbitrary waveform, provided that its frequency content is significantly below the resonant frequency of the system.

The magnetic fields produced by both TMS and RF ablations are strong enough to completely destroy the MR signal and, in the case of RF ablation, induce temperature changes, which also produce phase changes in the image. Hence, our strategy is to measure a greatly scaled down version (up to five orders of magnitude) of the electromagnetic field of interest, instead. Since it follows a linear relationship to the input current through the probe, the resulting magnetic field map can be re-scaled appropriately in order to calculate the actual treatment field. This approach allows image acquisition and also allows the operator to map the field before applying the treatment, in order to permit the appropriate position adjustments before without affecting unwanted tissue regions.

The scope of this article is limited to introducing the theory and demonstrating proof of concept for the method in a 7T small animal scanner. To that end, we imaged oscillating magnetic fields generated by a figure-eight coil inside simple phantoms over a range of frequencies and intensities. We defer characterization of the fields generated by TMS and/or RF thermal ablation probes in humans for future studies.

\section{Theory}

We begin by noting that in a typical MRI experiment, as soon as the spins' magnetization vector is tipped onto the transverse plane by application of a resonant radio frequency (RF) pulse (let's call that time $t_{0}$ ) turning on an additional magnetic field will cause the magnetization vector to gain phase relative to the rotating frame of reference as

$$
M_{x y}(\vec{r}, t)=M_{x y}\left(\vec{r}, t_{0}\right) \cdot e^{-i 2 \pi \gamma \int_{t_{0}}^{t} B_{z}(\vec{r}, \tau) d \tau} .
$$

Here, $M_{X X}(\vec{r}, t)$ and $B_{Z}(\vec{r}, t)$ are the magnetization vector and the additional magnetic field at position $r$ and time $t$, respectively, and $\gamma$ is the gyromagnetic ratio (the variable $\tau$ is used for time-integration). We note that the phase gain experienced by the magnetization vector is produced only by the component of the external magnetic field that lies along the direction of the scanner's main axis (let us designate it as the z-axis) and hence we focus on that component alone. The function describing the additional magnetic field is separable in space and time, i.e.,

$$
B_{z}(\vec{r}, t)=B_{z}^{\prime}(\vec{r}) f(t)
$$

where $f(t)$ is the waveform driving the device's magnetic field. The problem at hand consists of solving for the spatial map of an oscillating magnetic field, $B_{Z}{ }^{\prime}(r)$ generated by a device of interest given that its time course, $f(t)$, is known a priori and the magnetization vector field can be measured by the MRI experiment. We will refer to this field as the "external field" henceforth.

One approach to this problem, previously presented by our group (8), is to synchronize the driving function, $f(t)$, with pulse sequence's readout. In that case, the phase contribution 
from the k-space encoding gradients can be included into the well-known signal equation $(9,10)$, which can be modified as

$$
s(t)=\int M_{x y}\left(\vec{r}, t_{0}\right) \cdot e^{-i 2 \pi \gamma \int_{t_{0}}^{t} \vec{k}(\vec{r}, \rho)+B_{z}^{\prime}(\vec{r}) f(\tau) d \tau} d \vec{r}
$$

to include the effect of the external field. Recall that the term $\vec{k}(\vec{r}, \tau)$ indicates the signal's phase gain over time at each location due to the imaging pulse sequence's magnetic field gradients, also referred to as the ' $\mathrm{k}$-space trajectory'. By comparing the observed signals with and without the external field, one can solve for the spatial distribution of the external magnetic field, $B_{Z}{ }^{\prime}(\vec{r})$. While this equation can be solved using iterative algorithms, it is computationally expensive and not always stable in our experience.

On the other hand, if the device whose field we want to map can be pulsed during the interval between excitation and readout, the problem can be simplified significantly because the external field's contribution to the phase is no longer a function of time. The signal equation is now

$$
s(t)=\int M_{x y}\left(\vec{r}, t_{0}\right) e^{-i 2 \pi \gamma B_{z}^{\prime}(\vec{r}) F} \cdot e^{-i 2 \pi \gamma \int_{t_{0}}^{t} \vec{k}(\vec{r}, \tau) d \tau} d \vec{r}
$$

where $F$ is the time integral of the driving function $f(t)$ over the duration of the external magnetic pulse. In this case, the magnitude of the reconstructed image will not be affected, but its phase will be increased by

$$
\Delta \varphi(\vec{r})=2 \pi \gamma B_{z}^{\prime}(\vec{r}) F
$$

Calculating the spatial map of the external field, $B_{Z}{ }^{\prime}(\vec{r})$, is then straightforward. This approach is effective theoretically, as long as the induced phase, $\Delta \varphi$, is greater than $-\pi$ and less than $\pi$. Otherwise, phase wrapping may lead to erroneous results. While this procedure yields the component of the magnetic field along the direction of the scanner's main magnetic field, $B_{Z}{ }^{\prime}(\vec{r})$, it does not yield the other two Cartesian components of the vector field, $B_{x}^{\prime}(\vec{r})$ and $B_{y}^{\prime}(\vec{r})$. However, we have previously introduced a method to estimate the entire vector field by rotating both the object and the probe together inside the scanner and collecting several such projections. The details of the implementation can be found elsewhere (3), but the analysis can be summarized as follows. The object and probe are rotated together over several positions (minimum of three) and a Z-projection of the magnetic field of interest is collected, as described above.

Rigid body coregistration of the all images and the corresponding field projections is performed relative to a reference image. This can be carried out using any standard rigid body coregistration software (e.g., (11) (12)) that yields the rotation matrix in addition to the co-registered images. The entire magnetic field (i.e., all three Cartesian components) vector can then be calculated from the set of measured Z-projections at each orientation and the corresponding rotation matrices, as indicated in $(3,13)$.

\section{Methods}

All images were collected on a 7 Tesla scanner (7/310, Agilent Techonologies, Walnut Creek, CA) using a birdcage RF coil for excitation and acquisition. The first experiment was to verify our ability to image the magnetic field projections over a range of oscillating 
magnetic field intensities and frequencies. An angled figure-eight shape coil (loop diameter $=3 \mathrm{~cm}$, angle $\sim 130$ degrees) was constructed from magnet wire in order to mimic a TMS coil (e.g., MagVentures D-B80 coil, Magventure, Inc, Atlanta, Ga) and placed on a spherical phantom (diameter $=5 \mathrm{~cm}$ ) filled with water. The phantom-coil assembly was placed inside the scanner, oriented so that the bulk of the magnetic field produced by the coil was aligned with the main magnetic field of the scanner, as illustrated in Figure 1A. The coil was connected to an arbitrary function generator (HP 33120A function generator, Palo Alto, CA).

Ten slices were collected along the axial plane (or XY-plane in the magnet's frame of reference) using a standard gradient echo sequence $(\mathrm{TR}=116 \mathrm{~ms}, \mathrm{TE}=10 \mathrm{~ms}$, flip angle $=$ $20 \mathrm{deg}, \mathrm{FOV}=8 \mathrm{~cm}$, slice thickness $=2 \mathrm{~mm}$, matrix size $=128 \times 128, \mathrm{RF}$ and gradient spoiling were used to remove spurious magnetization). The magnetic field of interest was induced in the phantom by the probe during the acquisition of the gradient echo image signals. Specifically, a sine wave consisting of 3.25 cycles was transmitted through the probe immediately after the RF excitation pulse, but well before the frequency and phase encode gradients of the gradient echo sequence were turned on. Note that for this specific pulse was chosen because Equation 5, describing the phase gain created by the pulse, integrates elegantly to a linear function of the inverse of the pulse's frequency, $\omega$ :

$$
\Delta \varphi=2 \pi \gamma B_{z}^{\prime}(\vec{r}) \frac{1}{\omega} . \quad[6]
$$

The applied peak-to-peak voltages across the coil were $0,1,5,10$ and $14 \mathrm{~V}$ and their frequency was $0.5,1,5,10,50,100 \mathrm{kHz}$. In order to explore the voltage dependence in more depth, the experiment was repeated at $1 \mathrm{kHz}$ and $100 \mathrm{kHz}$ using a more finely sampled set of voltages (from 10 to $14 \mathrm{~V}_{\mathrm{pp}}$ at $0.5 \mathrm{~V}_{\mathrm{pp}}$ increments) but coarser image resolution $(64 \times 64)$ to reduce acquisition time. The current through the probe was verified to be constant at each voltage regardless of frequency, indicating that the probe's reactance (and hence, its inductance) was negligible over the frequency range of this work. The images were reconstructed and the phase difference due to the magnetic pulse was computed at each voltage and frequency.

A second experiment was carried out to test our ability to reconstruct the three dimensions of the oscillating magnetic vector field. A small phantom was constructed by filling a small bottle with gelatin and introducing a thin hollow tube only partially filled with gelatin and placed diagonally inside the gelatin, in order to give the phantom an asymmetric structure (see Figure 1B). A second figure-eight probe (6 turns on each loop, $2 \mathrm{~cm}$ diameter loops) was attached to the phantom and the whole assembly was placed inside the scanner. The object was imaged at seven different orientations using a gradient echo sequence $(\mathrm{TR}=60$ $\mathrm{ms}, \mathrm{TE}=4 \mathrm{~ms}$, flip angle $=30 \mathrm{deg}, \mathrm{FOV}=6.4 \mathrm{~cm}$, slice thickness $=1 \mathrm{~mm}$, matrix size $=64$ $\times 64 \times 50, \mathrm{RF}$ and gradient spoiling were used to remove spurious magnetization). The phantom/probe assembly was imaged at each position twice: without any current running through the probe and also while a current pulse was transmitted through the probe immediately after the excitation RF pulse. This pulse lasted $1.5 \mathrm{~ms}$ and consisted of three cycles (i.e., frequency $=2 \mathrm{KHz}$ ) of a DC-shifted cosine wave oscillating between 0 and 1 Volts.

The images were subsequently reconstructed and the phase difference between the complex images with and without the magnetic pulse were computed at each position. The projection of the magnetic field onto the Z-axis $\left(B_{Z}\right)$ was computed from these phase differences and from the integral of the applied magnetic pulse. Next, the images were coregistered by rigid body affine transformation using FLIRT (http://fsl.fmrib.ox.ac.uk/fsl/fslwiki/FLIRT). The 
resulting affine transformation matrices for each orientation were used to construct the projection matrix $\boldsymbol{P}$ using the method presented by Hernandez-Garcia et al. (3), which relates the observed projections, $B_{z}^{n}$, to the original magnetic field vector $B_{x y z}$ at every voxel by:

$$
\left[\begin{array}{c}
B_{z}^{1} \\
B_{z}^{2} \\
\vdots \\
B_{z}^{n}
\end{array}\right]=\boldsymbol{P}\left[\begin{array}{c}
B_{x} \\
B_{y} \\
B_{z}
\end{array}\right] \cdot \quad[7]
$$

The magnetic vector field, $\left[B_{X} B_{y} B_{Z}\right]^{T}$, at each voxel was then computed by inverting the projection matrix $\boldsymbol{P}$ using the least-squares pseudo-inverse and multiplying it by the vector of measured projections at that voxel. The resulting maps of the three Cartesian components were then used to construct vector field plots, which were overlaid on intensity maps.

\section{Results}

The first experiment demonstrates the dependence of the phase accrued due to the probe's magnetic field on the frequency and amplitude of the field. Images of the phase difference induced by the magnetic field pulses over the range of intensities (voltage across the probe) and frequencies from the probe at a plane $10 \mathrm{~mm}$ away from the coil's vertex can be seen in Figure 2. This location was chosen as it approximates a typical stimulation region in TMS experiments. There is a clear trend of increasing phase gain with lower frequency and higher voltage.

The average of the absolute phase difference over the object in the slice was fit to a simple linear function of the inverse of the frequency, in accordance to Equation 6 above. It is clear from Figure 3 that the phase changes do indeed follow a linear function of the inverse of the frequency and the magnetic field's amplitude. The individual correlation coefficients and their confidence intervals are shown in Table 1. All correlation coefficients between the phase gain and the inverse of the frequency were above 0.96. Also consistent with Equation 6 , all correlation coefficients between the phase gain and the peak-to-peak voltage amplitude were above 0.82 (see Table 1). More specifically, Figure 3A shows plots of the phase gain against the inverse frequency of the pulse and Figure 3B shows a plot of the fitted slopes from the frequency plots against the peak-to-peak voltage. The fitted slopes of the frequency dependence plots were plotted in turn against the voltage applied to the probe, again yielding a strong linear relationship ( $r>0.99$ with $95 \%$ confidence interval of 0.001$)$. Conversely, Figure $3 \mathrm{C}$ shows plots of the phase gain against the peak-to-peak amplitude voltage of the pulse and Figure 3D shows a plot of the fitted slopes from the voltage plots against the inverse frequency. The fitted slopes of the voltage dependence plots were in turn plotted against the inverse frequency of the applied to the probe, again yielding a strong linear relationship ( $r>0.99$ with $95 \%$ confidence interval of 0.001 ).

The Z-component (aligned with the magnet's main axis) of the magnetic fields induced by the probe at each frequency and voltage were computed from the above phase maps and can be seen in Figure 4. Consistent with expectation from basic electromagnetic theory, the induced magnetic field increases linearly with the current through the loop, (proportional to the applied voltage) but is largely independent of frequency in this regime. However, at the higher frequencies the images were notably noisier because the amount of phase gained was much smaller, hence yielding images with a smaller signal-to-noise-ratio. 
Magnetic vector field maps were reconstructed from the phase images collected in the second experiment. The three Cartesian components of the magnetic field inside the object are displayed as axial slices in Figure 5. Figure 6 displays three intersecting orthogonal views of that same vector field's magnitude on a log scale. Streamlines are overlaid on top of the magnitude images to indicate the direction of the magnetic field that resulted from the projection process. Note that the empty space apparent in Figures 5 and 6 inside the phantom is due to the partially filled hollow tube.

\section{Discussion}

We have presented a simple method to map oscillating magnetic fields inside tissue using MRI. This method is quite versatile in that it allows for imaging the spatial distribution of a broad spectrum of frequencies and does not require any additional hardware, except for the ability to synchronize the source of the magnetic field with the MR scanner, which can typically be achieved easily via TTL lines. The main constraints of the method are that (1) it requires control of the temporal characteristics of the magnetic field under scrutiny, (2) it can only be used at low field amplitudes (in the order of a few mGauss) so that the phase gain can be kept within 0 and $2 \pi$ radians, which means that in many cases we can image only a scaled down version of the field, (3) the frequency content of the waveform must be below the Larmor frequency of the tissue's spins. Phase wraps within the images may also constitute a challenge to this technique. This can be overcome by employing a number of numerical techniques to 'unwrap' the phase, such as those in $(1,14-16)$. If those techniques prove to be insufficient, though, one can also resort to collecting a second field map of that region at a lower power level.

Beyond these constraints, there are other practical issues that must be considered as well. The first one is that, in order to calculate all three components of the magnetic field, the object must be reoriented several times in the bore of the magnet while the probe in question remains fixed to the object. While this is somewhat cumbersome, we have demonstrated before that it is quite feasible because the changes in orientation can be subtle (in the range of $<10$ degree rotations) as long as a large number of orientations is acquired (see $(3,13)$ for a demonstration in a human head with a TMS device). Second, the probe itself can potentially distort the images because of ferrous materials in its construction. Thus, one must be careful in the choice of the imaging sequence. For example, one can use shorter echo times and faster readouts if using gradient echo imaging or the technique can be readily adapted as a spin-echo readout by pulsing the magnetic field under scrutiny in the period between the 90 degree and the 180 degree pulses of the MR pulse sequence.

Previous work by Bohning et al (2) showed that one can obtain a measurement of a TMS probe's magnetic field without collecting multiple projections of the field, in contrast to the present article as well as our previous work (3). While the single projection method is simpler to implement, it only yields the z-component of the magnetic field in question. In that implementation, the coil had to be positioned perpendicularly to the direction of the main axis of the field in order to measure the main part of the field. That approach also constrained the imaging region to the near field, where the magnetic field is mostly aligned perpendicularly to the probe. Further from the coil, however, as the magnetic field vector curves and diverges from the main axis, the results would be inaccurate. In our previous work with DC fields (3), we demonstrated that a minimum of three projections are needed under ideal conditions, but that the more projections are collected, the better the condition number of the projection matrix, and hence the easier it is to invert the matrix and solve for the whole vector. 
There are several potential biomedical applications for this technique. One family of applications is image guidance for transcranial magnetic stimulation (TMS) and radiofrequency ablation (RFA), which is highly desirable for targeting and dosimetry purposes (4,17-19). In both cases, the specificity and accuracy of the treatment are crucial but it is not straightforward to deliver the energy to the target without radiating unwanted tissue. Hence a great deal of effort has been spent on improving targeting capabilities through computer simulations (20-28) (29) or image guidance $(30)(3,31,32)$. The current technique yields an accurate map of the magnetic vector field in the target region. However, we note that the parameter of greatest interest in both RFA and TMS is the electric field, but knowledge of the magnetic field permits us to evaluate the electric field through Faraday's equation for induction. One strategy could be to calculate only the magnitude of electric field along a discrete contour, determined by the edges of each pixel, by scaling the magnitude of the magnetic field on the cross sectional area of that segment by the rate of change of the current pulse, according to

$$
|\oint \vec{E} \cdot d l|=\left|-\frac{d}{d t} \int \vec{B} \cdot d A\right|
$$

of the induced current density in the tissue, $\mathrm{J}$, is determined by

$$
|\vec{J}|=|\sigma \vec{E}| \quad[9]
$$

Lastly, another family of biomedical applications for this technique is mapping of the electrical properties of tissue (e.g., permeability, conductance, permittivity, etc.), which has recently received much attention as a potential diagnostic tool (33) (34) (35). In these applications, knowledge of the magnetic field can be used to solve for the tissue parameters of interest through electromagnetic theory.

\section{Acknowledgments}

The authors thank Jeremy Gam and Robert Welsh for their technical help and advice. This work was funded by a grant from the National Institutes of Health (NS058691).

\section{References}

1. Chen N, Wyrwicz A. Correction for EPI distortions using multi-echo gradient-echo imaging. Magn Reson Med. 1999; 41(6):1206-1213. [PubMed: 10371453]

2. Bohning D, Pecheny A, Epstein C, Speer A, Vincent D, Dannels W, George M. Mapping transcranial magnetic stimulation (TMS) fields in vivo with MRI. Neuroreport. 1997; 8(11):25352538. [PubMed: 9261822]

3. Hernandez-Garcia L, Lee S, Grissom W. An approach to MRI-based dosimetry for transcranial magnetic stimulation. Neuroimage. 2007; 36(4):1171-1178. [PubMed: 17509897]

4. Friedman M, Mikityansky I, Kam A, Libutti SK, Walther MM, Neeman Z, Locklin JK, Wood BJ. Radiofrequency Ablation of Cancer. CardioVascular and Interventional Radiology. 2004; 27(5): 427-434. [PubMed: 15383844]

5. Jezzard P, Balaban R. Correcting for geometric distortion in echo-planar images from Bo field distortions. Magnetic Resonance in Medicine. 1995; 34:65-73. [PubMed: 7674900]

6. Sacolick LI, Wiesinger F, Hancu I, Vogel MW. B1 mapping by Bloch-Siegert shift. Magnetic Resonance in Medicine. 2010; 63(5):1315-1322. [PubMed: 20432302]

7. Halpern-Manners NW, Bajaj VS, Teisseyre TZ, Pines A. Magnetic resonance imaging of oscillating electrical currents. Proceedings of the National Academy of Sciences. 2010; 107(19):8519-8524. 
8. Bhatia, VR.; Hernandez-Garcia, L. Oscillating Magnetic Field Mapping using MRI. Montreal, Quebec, Canada: 2011.

9. Macovski, A. Medical imaging systems. Vol. xiv. Englewood Cliffs, N.J: Prentice-Hall; 1983. p. 256

10. Haacke, EM. Magnetic resonance imaging: physical principles and sequence design. Vol. xxvii. New York: Wiley; 1999. p. 914

11. Friston KJ, Ashburner J, Frith CD, Poline JB, Heather JD, Frackowiak RSJ. Spatial registration and normalization of images. Human Brain Mapping. 1995; 3(3):165-189.

12. Snyder, AZ.; Myer, R.; Cunningham, VJ.; Bailey, D.; Jones, T. Quantification of Brain Functioning Using PET. San Diego: Academic Press; 1996. Difference image versus ratio image error function forms in PET-PET realignment; p. 131-137.

13. Lee, S.; Hernandez-Garcia, L.; Grissom, W. MRI based measurement of magnetic field distribution generated by transcranial magnetic stimulation coils. Seattle, WA: 2006 May.

14. Dong Y, Ji J. Phase unwrapping using region-based markov random field model. Conference proceedings: Annual International Conference of the IEEE Engineering in Medicine and Biology Society IEEE Engineering in Medicine and Biology Society Conference. 2010; 2010:3309-3312. [PubMed: 21096819]

15. Langley J, Zhao Q. A model-based 3D phase unwrapping algorithm using Gegenbauer polynomials. Physics in Medicine and Biology. 2009; 54(17):5237-5252. [PubMed: 19671967]

16. Liang ZP. A model-based method for phase unwrapping. IEEE Transactions on Medical Imaging. 1996; 15(6):893-897. [PubMed: 18215968]

17. Cardinal J, Klune JR, Chory E, Jeyabalan G, Kanzius JS, Nalesnik M, Geller DA. Noninvasive radiofrequency ablation of cancer targeted by gold nanoparticles. Surgery. 2008; 144(2):125-132. [PubMed: 18656617]

18. Crocetti L, Lencioni R, DeBeni S, See TC, Pina CD, Bartolozzi C. Targeting Liver Lesions for Radiofrequency Ablation: An Experimental Feasibility Study Using a CT-US Fusion Imaging System. Investigative Radiology. 2008; 43(1):33-39.10.1097/RLI.1090b1013e31815597dc [PubMed: 18097275]

19. Gazelle GS, Goldberg SN, Solbiati L, Livraghi T. Tumor Ablation with Radio-frequency Energy1. Radiology. 2000; 217(3):633-646. [PubMed: 11110923]

20. Cerri G, De Leo R, Moglie F, Schiavoni A. An accurate 3-D model for magnetic stimulation of the brain cortex. J Med Eng Technol. 1995; 19(1):7-16. [PubMed: 7562982]

21. Roth Y, Amir A, Levkovitz Y, Zangen A. Three-dimensional distribution of the electric field induced in the brain by transcranial magnetic stimulation using figure- 8 and deep H-coils. J Clin Neurophysiol. 2007; 24(1):31-38. [PubMed: 17277575]

22. Ruohonen J, Ilmoniemi R. Modeling of the stimulating field generation in TMS. Electroencephalogr Clin Neurophysiol Suppl. 1999; 51:30-40. [PubMed: 10590933]

23. Salinas FS, Lancaster JL, Fox PT. 3D modeling of the total electric field induced by transcranial magnetic stimulation using the boundary element method. Physics in Medicine and Biology. 2009; 12:3631-3647. [PubMed: 19458407]

24. Thielscher A, Kammer T. Electric field properties of two commercial figure-8 coils in TMS: calculation of focality and efficiency. Clinical neurophysiology: official journal of the International Federation of Clinical Neurophysiology. 2004; 115(7):1697-1708. [PubMed: 15203072]

25. Toschi N, Welt T, Guerrisi M, Keck ME. A reconstruction of the conductive phenomena elicited by transcranial magnetic stimulation in heterogeneous brain tissue. Phys Med. 2008; 24(2):80-86. [PubMed: 18296093]

26. Toschi N, Welt T, Guerrisi M, Keck ME. Transcranial magnetic stimulation in heterogeneous brain tissue: clinical impact on focality, reproducibility and true sham stimulation. J Psychiatr Res. 2009; 43(3):255-264. [PubMed: 18514227]

27. Jalinous R. Technical and practical aspects of magnetic nerve stimulation. J Clin Neurophysiol. 1991; 8(1):10-25. [PubMed: 2019644] 
28. De Leo R, Cerri G, Balducci D, Moglie F, Scarpino O, Guidi M. Computer modelling of brain cortex excitation by magnetic field pulses. J Med Eng Technol. 1992; 16(4):149-156. [PubMed: 1433245]

29. Zangen A, Roth Y, Voller B, Hallett M. Transcranial magnetic stimulation of deep brain regions: evidence for efficacy of the H-Coil. Clinical Neurophysiology. 2005; 116(4):775-779. [PubMed: 15792886]

30. Bohning D, Shastri A, McConnell K, Nahas Z, Lorberbaum J, Roberts D, Teneback C, Vincent D, George M. A combined TMS/fMRI study of intensity-dependent TMS over motor cortex. Biol Psychiatry. 1999; 45(4):385-394. [PubMed: 10071706]

31. Bohning D, Shastri A, Wassermann E, Ziemann U, Lorberbaum J, Nahas Z, Lomarev M, George M. BOLD-f MRI response to single-pulse transcranial magnetic stimulation (TMS). J Magn Reson Imaging. 2000; 11(6):569-574. [PubMed: 10862054]

32. Bohning D, Denslow S, Bohning P, Walker J, George M. A TMS coil positioning/holding system for MR image-guided TMS interleaved with fMRI. Clin Neurophysiol. 2003; 114(11):2210-2219. [PubMed: 14580621]

33. Joy M, Nachman A, DeMonte T, Dinghui W, Weijing M. Electrical conductivity imaging using MRI measurement of the magnetic field vector. Sep 3-6.2009 2009:3158-3161.

34. Zhang X, Zhu S, He B. Imaging electric properties of biological tissues by RF field mapping in MRI. IEEE Transactions on Medical Imaging. 2010; 29(2):474-481. [PubMed: 20129847]

35. Haacke EM, Petropoulos LS, Nilges EW, Wu DH. Extraction of conductivity and permittivity using magnetic resonance imaging. Physics in Medicine and Biology. 1991; 36(6):723. 


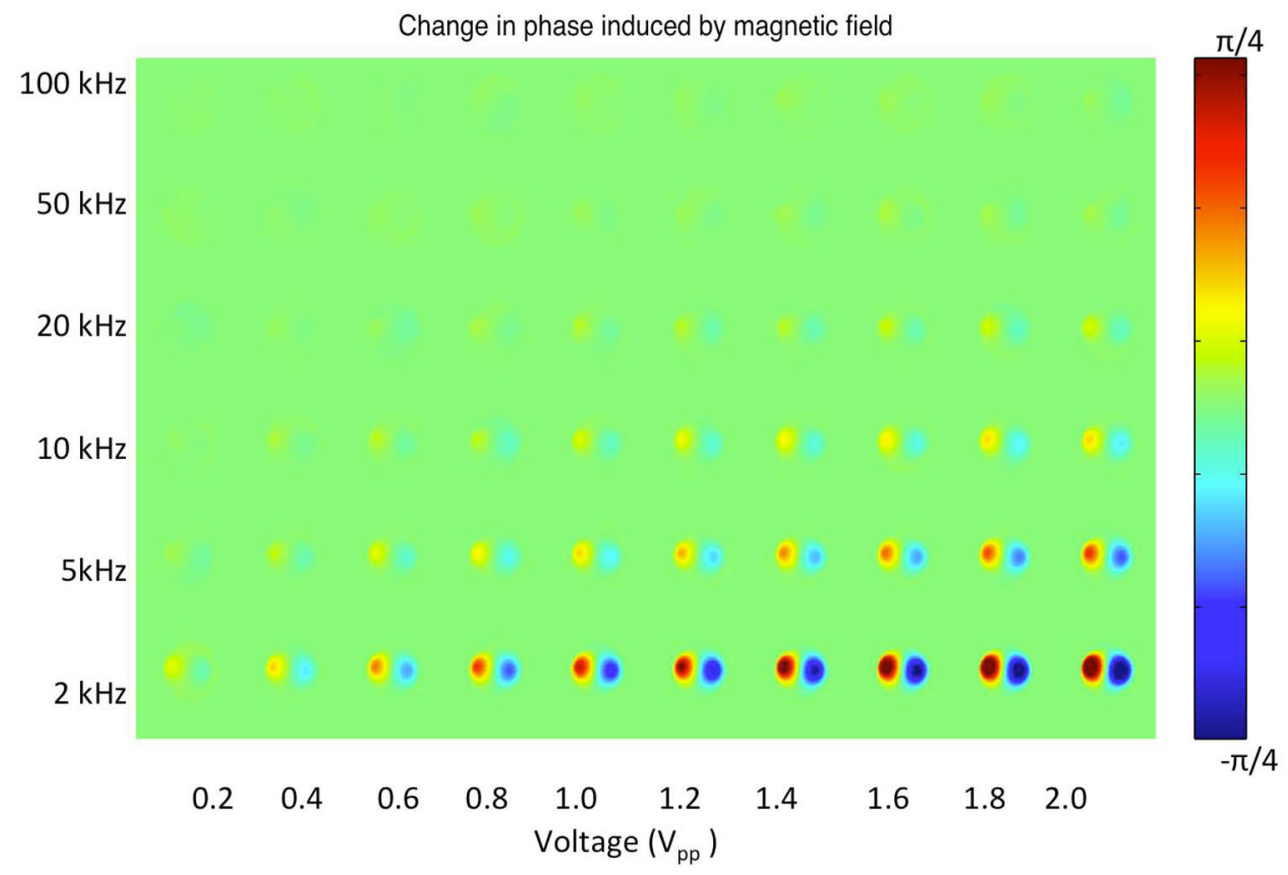

Figure 1.

Figure 1A: Experimental setup for measuring the relationship between frequency, magnetic field strength and signal phase gain using a simple figure-eight probe.

Figure $1 \mathrm{~B}$ - The agar phantom used in the second experiment with the angled figure-eight coil assembly attached to it. A hollow tube inside the phantom provided sufficient structural features for coregistration algorithms. 


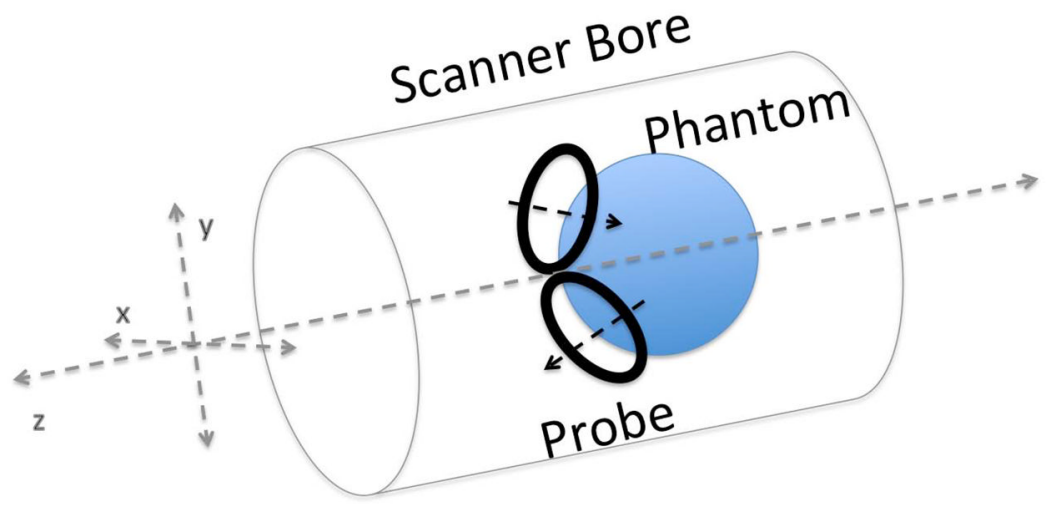

Figure 2.

Phase gain induced in a single slice of the phantom by a magnetic pulse generated by the probe depicted in Figure 1. The experiment was repeated over a range of frequencies and voltages applied to the probe. 
(A) Frequency Dependence

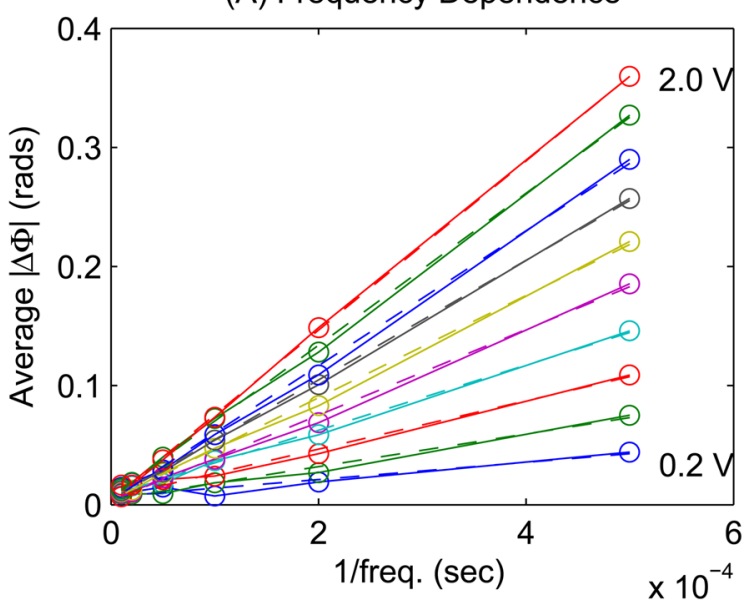

(C) Voltage Dependence

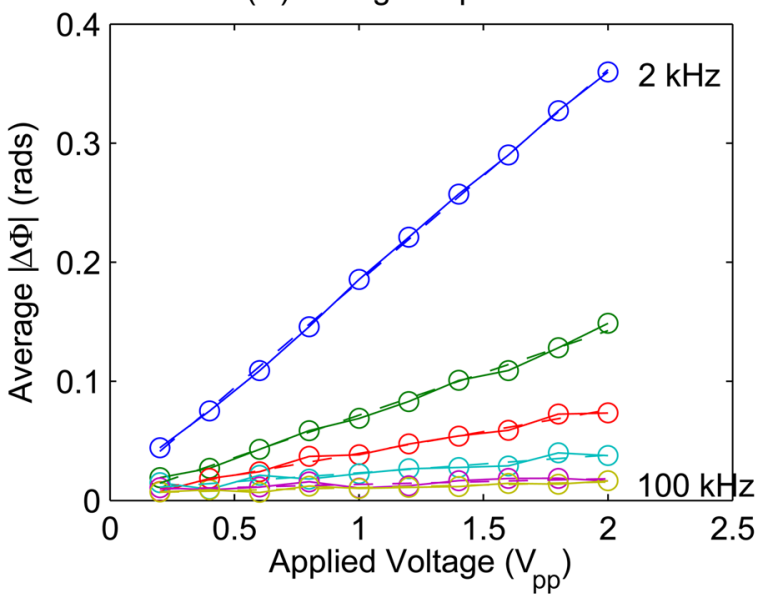

(B) Slopes

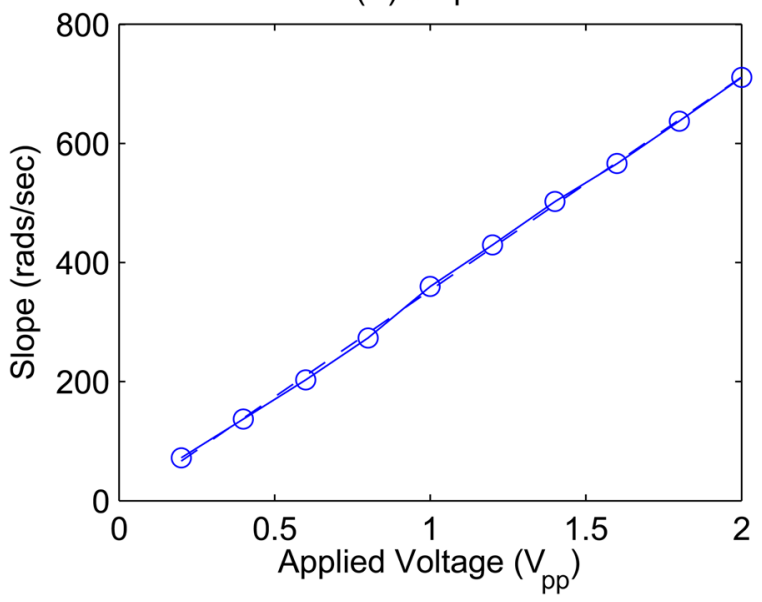

(D) Slopes

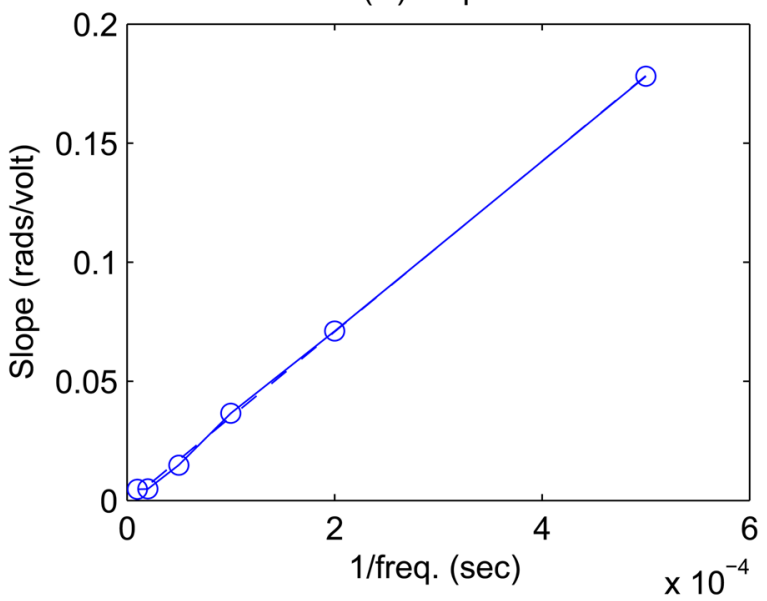

Figure 3.

Dependence of the phase gain on the frequency and voltage amplitude of the pulses. Panel A shows the dependence of the phase gain on the inverse of the frequency over a range of voltage amplitudes. Consistent with Equation 6, Panel B shows that the slopes of these plots are also linearly dependent on the magnetic field, and hence the applied voltage. Conversely, panel $\mathrm{C}$ shows the linear dependence of the phase gain on the voltage amplitude and panel $\mathrm{D}$ indicates the linear relationship between the slopes of the plots in panel $\mathrm{C}$ and the inverse frequency of the pulse. 


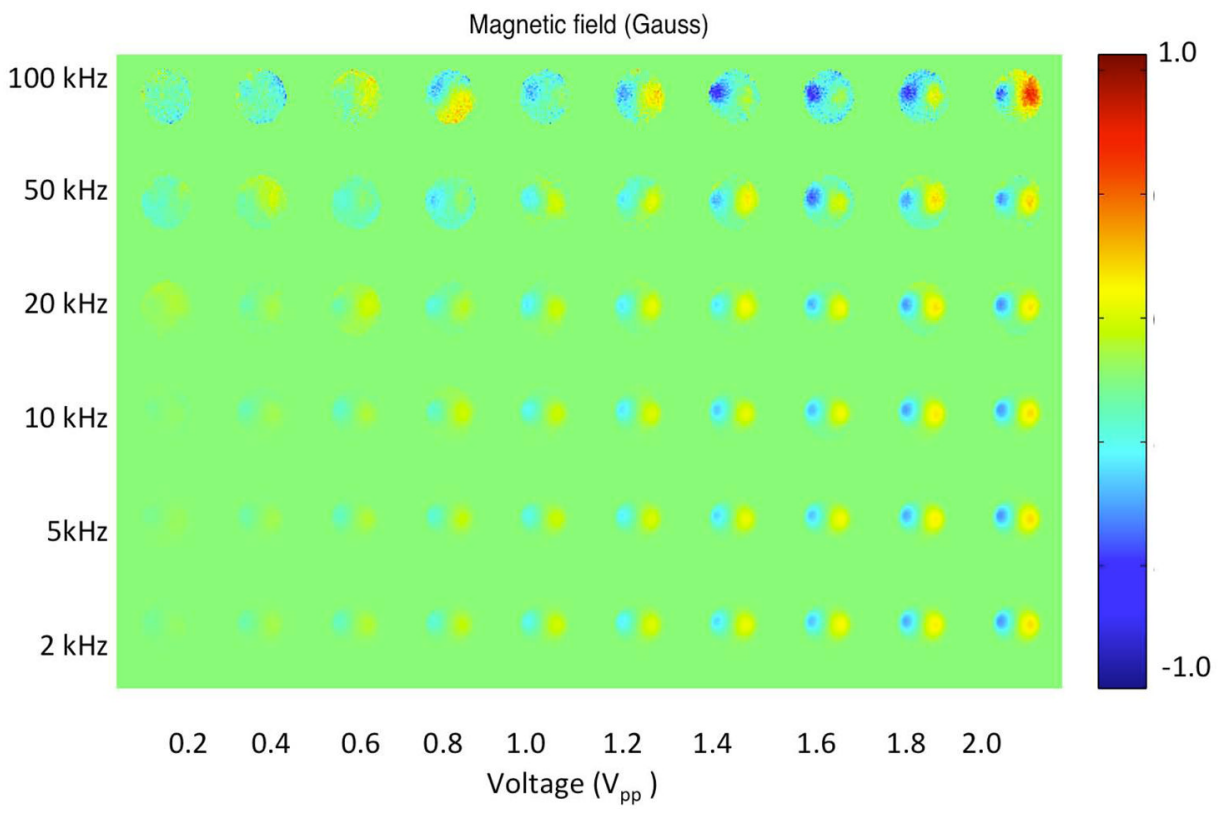

Figure 4.

Maps of the Z-component of magnetic field induced by a sinusoidal pulse through the probe in Figure 1. The frequency and peak-to-peak voltage were varied as indicated in the plot. The field was calculated using the relationship in Equation 5. 


\section{Cartesian Components of the Magnetic Field Generated by the Probe}
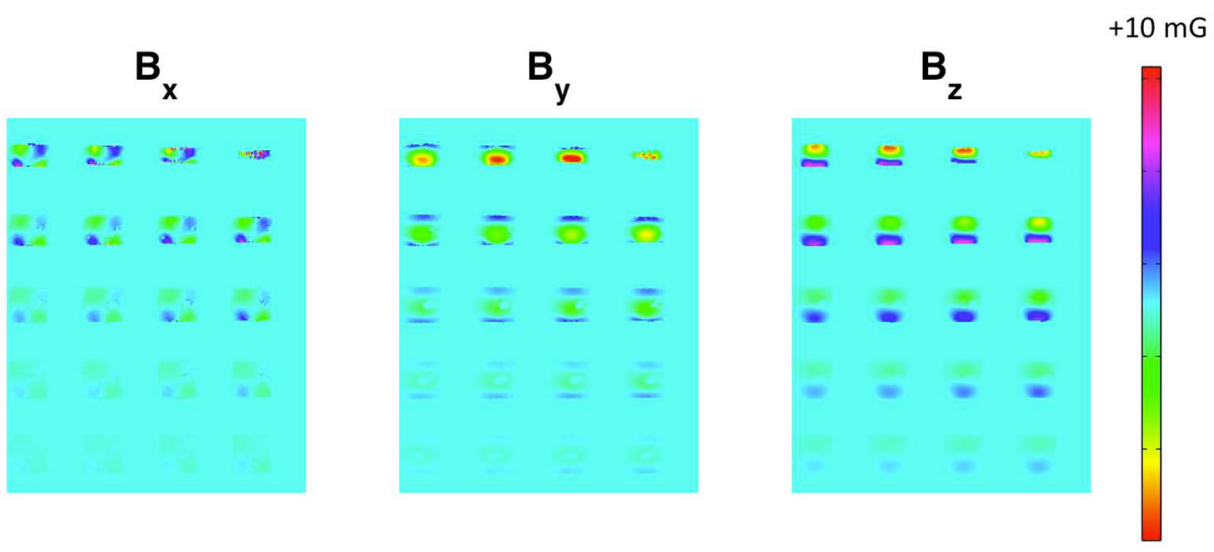

$-10 \mathrm{mG}$

Figure 5.

Axial images of the three Cartesian components of the magnetic field vector generated by the angled figure-eight probe on an agar phantom. 


\section{Cross-Sectional Views of Magnetic Vector Field Generated by the Probe}
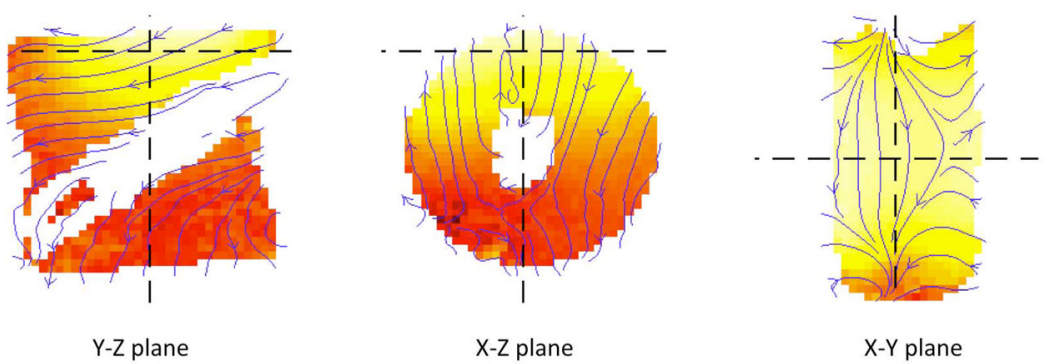

Figure 6.

Three orthogonal views of the magnetic field displayed in Figure 5. In this figure, the field's magnitude is displayed in a log scale to aid visualization of the dynamic range. Streamline arrows are overlaid on top of the magnitude maps in order to indicate the directionality of the vector field (note that the empty space inside the phantom is a hollow tube, inserted to provide the phantom enough physical features for coregistration). The black cross-hairs indicate the intersection point of the three planes depicted. 


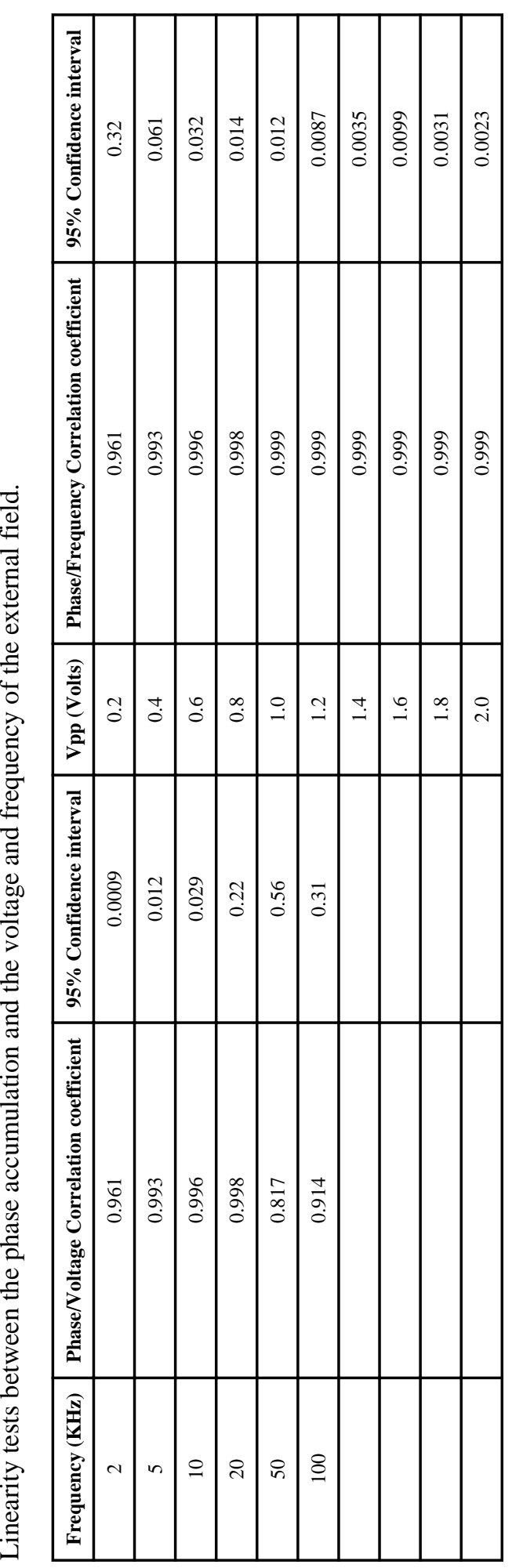

NMR Biomed. Author manuscript; available in PMC 2014 June 01. 